Relations industrielles

Industrial Relations

\title{
Le texte de la convention collective et les usages
}

\section{Pierre Verge}

Volume 22, numéro 3, 1967

URI : https://id.erudit.org/iderudit/027817ar

DOI : https://doi.org/10.7202/027817ar

Aller au sommaire du numéro

\section{Éditeur(s)}

Département des relations industrielles de l'Université Laval

\section{ISSN}

0034-379X (imprimé)

1703-8138 (numérique)

Découvrir la revue

\section{Citer cet article}

Verge, P. (1967). Le texte de la convention collective et les usages. Relations industrielles / Industrial Relations, 22(3), 446-449.

https://doi.org/10.7202/027817ar
Résumé de l'article

Un arbitre ${ }^{1}$ se fonde sur la supériorité du texte de la convention pour refuser de donner effet à une pratique antérieure, mais contraire à celle-ci. Est-ce à nier la survie de tout usage pendant la durée de l'entente?

(1) Me Harold Lande, dans l'affaire Commission Hydroélectrique de Québec c. Le Syndicat canadien de la fonction publique (957), décision du 1er mars 1967, rapportée à (1967) R.DT. p. 184.
Tous droits réservés (C Département des relations industrielles de l'Université Laval, 1967
Ce document est protégé par la loi sur le droit d'auteur. L'utilisation des services d'Érudit (y compris la reproduction) est assujettie à sa politique d'utilisation que vous pouvez consulter en ligne.

https://apropos.erudit.org/fr/usagers/politique-dutilisation/ 
C'est déjò un fait accompli aux Etats-Unis où le Labor-Management Reporting and Disclosure Act of 1959 „ prévoit des conditions précises pour la mise en séquestre:

"SEC. 302. Trusteeships shall be established and administered by a labor orgonizotion over a subordincte body cnly in occordance with the constitution and bylows of the organization which has assumed trusteeship over the subordinate body and for the purpose of correcting corruption or financial malpractice, assuring the performance of collective bargaining agreements or other duties of a bargaining representotive, restoring democratic procedures, or otherwise carrying out the legitimate objects of such labor organization. $\infty$

Bien sûr qu'un simple texte de loi ne peut suffire pour assurer le bon gouvernement des hommes mais, n'est-ce pas lò un outil utile pour atteindre cet objectif?

"The law cannot create the spirit of self-government. It cannot compel union members to attend meetings or hold their officers to a strict accounting. It cannot compel members to see in labor unions scmething more than service organizations hired to obtain benefits in return for dues. The most the law can do is to secure the opportunity for workers who wish to take an active part in democratic unions without undue loss of personol freedom. In my opinion careful legislation securing union membership to employees in bargaining units, guaranteeing fair election, and curtailling the duration of trusteeships would increase the opportunity without weakening our labor unions or impairing their independence." (Archibald Cox, Harvard Law Review, vol. 72, No. 4, «The role of law in preserving union democracy $\boldsymbol{\text { s.) }}$

\title{
LE TEXTE DE LA CONVENTION COLLECTIVE ET LES USAGES
}

Pierre Verge

\author{
Un arbitre ${ }^{1}$ se fonde sur la supériorité du texte de la convention pour \\ refuser de donner effet ò une pratique antérieure, mais contraire d̀ celle-ci. \\ Est-ce ò nier la survie de tout usage pendant la durée de l'entente?
}

\section{Les faits et la décision.}

Avant la signature de la convention collective, l'employeur exigeait qu'une certaine catégorie de salariés soient "disponibles vingt-quatre heures par jours, à tour de rôle, en cas d'urgence $»$, ceci sans rémunération, mais sous peine de renvoi

Neuf jours après la conclusion de cette première entente collective, le syndicat prétend, à l'occasion d'un grief, que cette pratique se trouve désormais abolie. La convention n'en fait pas mention; or, selon le syndicat, elle « contient toutes les conditions de travail et celles qui ne sont pas mentionnées ne peuvent plus être considérées comme conditions de travail . Au surplus, l'usage dont il s'agit va, prétend-il, à l'encontre d'une disposition de la convention venant établir la durée de la journée et de la semaine de travail.

(1) Me Harold Lande, dans l'affaire Commission Hydroślectrique de Québec c. Le Syndicot canadien de la fanction publique (957), décision du ler mars 1967, rapportée à (1967) R.DT.p. 184 
L'employeur répond à ce dernier argument en faisant une distinction entre la durée du travail et la période de disponibilité s. II signale aussi que l'on n'a pas retenu dans lo convention un texte que le syndicat avait d'abord voulu y insérer ò l'effet d'interdire à l'employeur de requérir que certains employés restent disponibles au-delò des heures de travoil. Fondamentalement, il expose enfin: "L'obligation de disponibilité o toujours fait partie du travail régulier et habituel des techniciens; pour que cette pratique soit abolie par lo convention, il aurait fallu que ce fût fait soit d'une façon explicite cu par des clauses contradictoires

L'arbitre est d'avis que le grief est bien-fondé:

* Dans mon opinion, lo convention écrite remplace toutes les conditions qui existaient antérieurement y compris le droit d'exiger la « disponibilité ». Tant qu'il n'y a pas de convention collective, l'employeur conserve tous les droits $q u^{\prime} i l$ a déjà. Une fois qu'il a signé une convention collective, il ne peut exercer les droits qu'il avait ovant en outant qu'ils ne sont pas limités, diminués ou éliminés par la convention. La convention, une fois signée, est la seule loi qui existe entre les parties, et je ne puis juger des droits et des obligations des parties que par ce qui est écrit dans la convention. L'art. 19.01 est très clair. Il détermine explicitement les heures durant lesquelles les hommes doivent travailler soit de 8.30 a.m., à 4.45 p.m., chaque jour. Après 4.45 p.m., selon les termes de la convention écrite, les hommes sont libres de faire comme bon leur semble et ne sont plus sous la juridiction de la compagnie. (1967, R.D.T., p. 189.)

II mentionne également que si la convention se préoccupe expressément de la survie de certaines pratiques antérieures, elle est par contre muette à l'endroit de l'obligation de disponibilité et il conclut:

- Lo compognie ne peut référer à la protique établie lorsque le contrat luimême est ambigu et qu'il requiert une interprétation. Or, le texte de l'art. 19.01 est clair et non équivoque et, par conséquent, on ne peut admettre de référer à la pratique établie. Si on le faisait, on changerait la convention écrite et l'arbitre ajouterait une nouvelle condition dans la convention ce qui est contraire à l'art. 13.04 de ladite convention:

- L'arbire n'a aucune juridiction pour ajouter, soustraire, modifier ou amender quoi que ce soit dans cette convention. (1967, R.D.T. P. 191.)

\section{COMMENTAIRE:}

La survie de l'usage pendant la durée de la convention ne peut manquer de soulever des considérations relatives à la nature même de cette dernière. Ainsi, peut-on notamment faire état du caractère documentaire $(A)$ et bilatéral $(B)$ de la convention.

A) L'usage et le caractère documentaire de la convention collective. ....L'usage, certes, peut avoir le mérite de représenter un aspect de la réalité dans l'entreprise, aspect que ne traduit pas l'entente écrite. Celle-ci doit, en effet, parfois se contenter de s'exprimer en termes fort généraux. Elle a toutefois pour elle la valeur d'un accord solennel. D'où ces règles d'interprétation succinctes:

- Si ... la convention collective est muette ou plutôt ambiguë sur un point qui d'outre part fait l'objet d'un usage clairement démontré, nous posons comme premier principe qu'un conseil d'arbitrage doit respecter cet usage comme s'il était inscrit dans une convention. collective écrite, et contraindre au besoin les deux parties à n'y pas toucher durant la durée de la convention en cours. 
Si d'outre part on oppose un usage mal démontré (et qui est plutôt une pratique sporadique) à un texte de convention peu clair ou fort clair, c'est la convention qui évidemment, l'emportero dons le jugement des orbitres.

Si enfin on met en opposition un usage mal prouvé et un texte de convention ambigu, ou encore un usage parfaitement démontré et une stipulation écrite parfaitement limpide, c'est lo convention écrite qui l'emportero oux yeux des arbitres, à cause du caractère plus sûr, plus précis et plus solennel de cette dernière. ${ }^{2}$ biables.

Les positions de l'arbitre, dans l'espèce que nous onalysons, sont en substance sem-

II admet, d'une part, que l'usage puisse servir à interpréter une disposition ambigue de la convention 3 ; dons l'espèce, toutefois, celle-ci stipule clairement la durée maximale du travail. D'autre part, il se trouve à fonder sa décision de faire prévaloir le texte de la convention sur l'usage ontérieur, sur l'opposition qui se trouve ò exister entre cette cisposition, qui énonoce la durée maximale du travail, et la protique de l'employeur.

L'on tire donc pleinement les conséquences découlant du caractère documentaire de la convention; sans doute l'espèce ne permettait-elle pas d'insister davantage sur son coractère bilatáral..

\section{B) L'usage antérieur et le coroctère bilatéral de lo convention.}

Essentiellement, il fout ici répondre à la question suivante: les conditions de travail \$, auxquelles l'arbitre doit donner plein effet, se trouvent-elles énoncées de façon limitative dans le texte de la convention? L'usage, en d'autres termes, s'il peut servir à interpréter la convention, mais s'il ne peut la contredire, peut-il être lui-même générateur de conditions de travail et ajouter ainsi à celles énoncées dans le texte de la convention?

Il y a ici lieu d'envisager successivement le cos de l'usage qui s'est établi avant la convention et celui de l'usage qui a pris racine pendant sa durée.

L'espèce faisoit montre d'une protique établie unilatérolement par l'employeur ovant ia signature d'une première convention. L'arbitre fait droit ou grief du motif de la contradiction qui se présentait entre la pratique de l'employeur de requérir la e disponibilité , et le texte de lo convention relatif à lo durée du trovail. II laisse néanmoins clairement entendre qu'il est aussi fovorable à la prétention du syndicat à l'effet que lo convention contient toutes les conditions de travail: Dons mon opinion, dit-il, la convention remplace toutes les conditions qui existaient ontérieurement, y compris le droit d'exiger la a disponibilité . Seule une mention expresse dans la convention prévoyant la survie de l'usage antérieure eût donc permis celle-ci, mais cette mention n'existait pas.

(2) Règles énoncées par monsieur Roger Chartier, dans une décision majoritaire reproduite à (1959) 14, Relations industrielles, p. 92.

(3) Dans une outre décision récente, Me Lande ovait d'ailleurs eu l'occosion de recourir ¿ un usage établi pour clarifier le texte d'une convention. Voir: Le Syndicat national des travailleurs en produits chimiques de Shawinigan (C.S.N.) c. Shawinigan Chemicals Limited, (1967), R.D.T., 56. Voir également: Domtar Newsprint Limited c. Le Syndicat national de la division forestière d'East Angus et Windsor et al., décision de monsieur le juge JeanLouis Peloquin en date du 19 mai 1965, rapportée dans Décisions sur les conflits de droit dans les relations de travail, no 303-2. 
S'ogissont d'une protique établie unilatéralement por l'employeur avant la convention, cette prise de position rend pleinement justice à l'avènement du stade des relations collectives dans l'entreprise: The change from individual to Collective Bargaining is o change of kind and not merely a difference in degree. The introduction of a Collective Borgaining regime involves the occeptance by the parties of assumptions which ore entirely alien to on era of individual bargaining. Hence, any ottempt to measure rights and duties in employer-employee relations by reference to pre-collective bargaining standards is an ottempt to re-enter o world which has ceased to exist "

Ahais, que dire d'un tel refus de donner effet à un usage, dans le cas, cette fois, où celui-ci a pris naissance après l'établissement de relations collectives entre l'employeur et le représentont de ses salariés, c'est-d-dire, essentiellement, pendant la durée de la convention? (L'espèce n'illustroit pos cette dernière hypothèse).

II fout d'abord constater que ce refus continuerait de s'imposer. Lo plupart du temps, en effet, la convention, comme dans le cas présent, stipule que l'arbitre n'a aucune juridiction pour ajouter quoi que ce soit... s. Même si elle ne le foit pos, la solution doit hobituellement être la même, selon les textes du Code du trovail: l'arbitre des griefs (a.88) n'a charge que de connaitre les emésententes relatives à l'interprétation ou do l'opplication d'une convention collective, (o.l,g), convention que l'on définit por ailleurs restrictivement comme étant "une entente écrite relative aux conditions de travail... $($ a.l, e)

Ce refus de donner effe d̀ l'usage, dans cette hypothèse, n'en serait pos, de ce fait, tellement opportun. L'arbitre ne se trouverait-il pos alors d̀ fermer les yeux sur une partie des relations entre l'employeur et le représentont de ses saloriés, en les réduisont ainsi ò la lettre du texte?

(4) Loskin, Boro, in re Peterboro Lock Mfg., Co. Ltd. (1953) 4, Lob. Arb. Cas. 1502. 